\title{
Petasites japonicus bakkenolide B inhibits lipopolysaccharide-induced pro-inflammatory cytokines via AMPK/Nrf2 induction in microglia
}

\author{
SUN YOUNG PARK ${ }^{1}$, MIN HYUN CHOI ${ }^{2}$, GEUNTAE PARK ${ }^{3}$ and YOUNG-WHAN CHOI ${ }^{2}$ \\ ${ }^{1}$ Bio-IT Fusion Technology Research Institute, Pusan National University, Busan, South Gyeongsang 46241; \\ ${ }^{2}$ Department of Horticultural Bioscience, Pusan National University, Miryang, South Gyeongsang 50463; \\ ${ }^{3}$ Department of Nanomaterials Engineering, Pusan National University, Busan, South Gyeongsang 46241, Republic of Korea
}

Received August 22, 2017; Accepted December 21, 2017

DOI: 10.3892/ijmm.2017.3350

\begin{abstract}
Abnormal neuroinflammatory responses have diverse roles in neuronal death, oxidative stress and neurodegenerative diseases, such as Alzheimer's and Parkinson's disease. Microglia regulate these responses via molecular signaling cascades that involve inflammatory cytokines and complement proteins. Bakkenolide B from Petasites japonicus exhibits significant anti-inflammatory and anti-allergic bioactivities. The present study investigated the anti-neuroinflammatory effects and underlying molecular mechanisms of bakkenolide B on the lipopolysaccharide (LPS)-mediated neuroinflammatory response in microglia. The results indicated that bakkenolide B pretreatment significantly reduced microglial production of interleukin (IL)-1 $\beta$, IL-6, IL-12, and tumor necrosis factor (TNF)- $\alpha$. Furthermore, this effect was associated with reduced production of reactive oxygen species. The role of bakkenolide B was then evaluated in the upregulation of nuclear factor erythroid 2-related factor 2 (Nrf2)/antioxidant response element (ARE) signaling pathways. The results suggested that bakkenolide B significantly upregulated Nrf2/ARE pathway-related downstream factors, such as NADPH dehydrogenase quinone-1 (NQO-1) and heme oxygenase-1 (HO-1). Silencing of Nrf2, HO-1 and NQO-1 diminished the anti-neuroinflammatory properties
\end{abstract}

Correspondence to: Professor Geuntae Park, Department of Nanomaterials Engineering, Pusan National University, 2 Busandaehak-ro 63beon-gil, Busan, South Gyeongsang 46241, Republic of Korea

E-mail: gtpark@pusan.ac.kr

Professor Young-Whan Choi, Department of Horticultural Bioscience, Pusan National University, 1268-50 Samrangjin-ro Samrangjin-eup Miryang, South Gyeongsang 50463, Republic of Korea

E-mail:ywchoi@pusan.ac.kr

Key words: microglia, bakkenolide B, anti-neuroinflammation, AMP-activated protein kinase, nuclear factor erythroid 2-related factor 2 of bakkenolide B. AMP-activated protein kinase (AMPK) activates the Nrf2/ARE signaling pathway, and the results of the present study demonstrated that bakkenolide B increased AMPK phosphorylation in microglia. In addition, an AMPK inhibitor abolished the bakkenolide B-induced increase in nuclear Nrf2, NQO-1 and HO-1 protein expression. Finally, an AMPK inhibitor diminished the bakkenolide B-mediated inhibition of LPS-stimulated TNF- $\alpha$ production. Taken together, the present results demonstrate that bakkenolide B may be an effective and therapeutically relevant AMPK/Nrf2 pathway activator for suppressing abnormal neuroinflammation in neurodegenerative diseases.

\section{Introduction}

Microglia have a tissue macrophage lineage and constitute the innate immune system of the central nervous system. The activities of microglia are critical for various neuronal functions, including clearance of neuronal debris, tissue repair, synaptic plasticity, and neurotrophic factor release $(1,2)$. Microglia also release various cytokines and growth factors to facilitate repair of neurons affected by disordered CNS homeostasis. However, abnormal microglial activation is maladaptive. Many pro-neuroinflammatory cytokines and cytotoxic mediators, including interleukin (IL)-12, IL-1 $\beta$, IL-6, tumor necrosis factor (TNF)- $\alpha$ and reactive oxygen species (ROS) are induced in response to abnormal microglial activation. This abnormal induction is injurious to neurons and results in neurodegenerative disease (3-5). The proper maintenance of microglial activation is important for brain development and brain injury repair. Several studies have characterized the neuroinflammatory response that abnormal microglial activation induces $(6,7)$. Notably, this response promotes the development of neurodegenerative diseases, such as Huntington's, Alzheimer's and Parkinson's.

Microglia can be categorized into two types based on distinct patterns of activation: classical M1 microglia and the alternative M2 microglia. M1 microglia activate inflammatory cytokines, including inducible IL-12, IL-1 $\beta$, IL-6 and TNF- $\alpha$, and promote neuronal injury. In contrast, M2 microglia produce anti-inflammatory molecules that reduce 
inflammation and repair tissue injury (8-10). Microglia with an M2-like phenotype release anti-neuroinflammatory molecules, such as nuclear factor erythroid 2-related factor 2 (Nrf2), NADPH dehydrogenase quinone-1 (NQO-1) and heme oxygenase-1 (HO-1) (11). These multifunctional proteins are involved in neuronal defense and repair systems. Nrf2 is a transcription factor that is responsible for the activation of several phase II antioxidant enzymes. Under unstimulated conditions, the negative regulator Kelch-like ECH-associated protein 1 (Keap1) maintains Nrf2 in the cytoplasm, which results in its ubiquitination and proteasomal degradation. During electrophilic stress, Keap1 is altered and Nrf2 translocates to the nucleus, binds to the antioxidant response element (ARE) promotor sequence, and activates the transcription of its target genes, including HO-1 and NQO1 $(12,13)$. Genetic and pharmacological studies indicate that both NQO1 and HO-1 provide neuroprotection. For example, cell culture studies have demonstrated that NQO1 and HO-1 overexpression in neuronal cells decreases oxidative damage following exposure to amyloid ßor glutamate. Consequently, microglia from HO-1 and NQO1-overexpressing transgenic mice are more resistant to lipopolysaccharide (LPS)-mediated and oxidative injury (14).

AMP-activated protein kinase (AMPK) is a family of intracellular serine/threonine protein kinases that serve roles in context-specific metabolism in response to metabolic stress, such as oxidative stress and neuroinflammation (15). Growing evidence suggests that AMPK activation prevents neuronal oxidative stress and damage under several pathological conditions. Furthermore, recent studies have demonstrated that AMPK is a negative modulator of neuroinflammatory responses and that AMPK has anti-neuroinflammatory properties in LPS-treated microglia. Thus, AMPK activation indicates an anti-neuroinflammatory response, regulates catabolism and anabolism and improves redox balance. AMPK also promotes microglial polarization towards the M2 phenotype $(16,17)$. Consistent with the emerging interplay between oxidative stress and neuroinflammation responses, recent studies suggest the possibility of cross talk between the AMPK and Nrf2/ARE pathways (18). A previous study from our group reported that AMPK signaling regulates microglia-induced Nrf2/ARE activation and that the AMPK inhibitor compound C suppressed neuroinflammation (19). Other studies have demonstrated that the inhibition of AMPK ameliorates the LPS-stimulated neuroinflammatory response through the inactivation of Nrf2/ARE signaling (20).

Natural compounds are gaining prominence as valuable candidate substrates for the development of medications. Bakkenolide B, the main constituent of Petasites japonicus leaves, is generally cultured in East Asia and used as both a vegetable and a folk remedy (21). P. japonicus has been used to treat various diseases, including headaches, chronic cough, fever, and asthma. A bioassay study has demonstrated that $P$. japonicus extracts and compounds have antioxidant, anti-inflammatory, and antitumor biological effects (22). P.japonicus bakkenolide B has been previously demonstrated by our group to exhibit both antiallergenic and anti-inflammatory effects (23). Furthermore, sesquiterpenoids from $P$. japonicus have neuroprotective properties in human neuroblastoma SH-SY5Y cells $(24,25)$. However, the effects of bakkenolide B on microglia-mediated neuroinflammatory activity have not been investigated. Thus, the present study investigated the effects of bakkenolide B on the microglial neuroinflammatory response. Additional mechanistic experiments verified that AMPK was associated with Nrf2/ARE activation. Taken together, the present results indicate that bakkenolide B may a potential candidate for treatment of abnormal neuroinflammatory-mediated neurodegenerative diseases.

\section{Materials and methods}

Reagents. CM-H2DCFDA was obtained from Thermo Fisher Scientific, Inc. (Waltham, MA, USA). Compound C, dimethyl sulfoxide (DMSO), MTT and other reagents were obtained from Sigma-Aldrich (Merck KGaA, Darmstadt, Germany). Dulbecco's modified Eagle's medium (DMEM) and fetal bovine serum (FBS) were obtained from Gibco (Thermo Fisher Scientific, Inc.). Antibodies for $\alpha$-tubulin [cat. no. sc-23948, 1:10,000 dilution, mouse immunoglobulin(Ig)G],TATA-binding protein (TBP, cat. no. sc-204, 1:500 dilution, rabbit IgG), inducible nitric oxide synthase (iNOS, cat. no. sc-8310, 1:1,000 dilution, rabbit $\operatorname{IgG}$ ), Nrf2 (cat. no. sc-722, 1:500 dilution, rabbit IgG), NQO1, (cat. no. sc-16464, 1:1,000 dilution, rabbit IgG) and HO-1 (cat. no. sc-10789, 1:1,000 dilution, rabbit IgG), as well as small interfering (si)RNAs against Nrf2 (cat. no. sc-37049), NQO1 (cat. no. sc-37140) and HO-1 (cat. no. sc-35555), were obtained from Santa Cruz Biotechnology, Inc. (Dallas, TX, USA). Antibodies against AMPK (cat. no. 2532, 1:1,000 dilution, rabbit IgG) and phosphorylated (p-) AMPK (cat. no. 2535, 1:1,000 dilution, rabbit IgG) were obtained from Cell Signaling Technology, Inc. (Danvers, MA, USA). FuGENE HD transfection reagent and $\mathrm{X}$-tremeGENE siRNA transfection reagent were obtained from Roche Diagnostics (Indianapolis, IN, USA). A cytotoxicity detection kit, measuring lactate dehydrogenase (LDH) activity, was purchased from Roche Diagnostics (Basel, Switzerland). A mouse Quantikine ELISA kit (for TNF- $\alpha$ cat. no. MTA00B; IL-1 $\beta$ cat. no. MLB00C; IL-6 cat. No M6000B and IL-12 cat. no. M1270) was acquired from R\&D Systems, Inc. (Minneapolis, MN, USA). All other basic experimental supplies and reagents were obtained from Sigma-Aldrich (Merck KGaA) and Invitrogen (Thermo Fisher Scientific, Inc.).

Extraction, isolation, and structure elucidation of bakkenolide $B$. Bakkenolide $\mathrm{B}$ was isolated using open column chromatography and its structure was previously elucidated by NMR spectroscopy (23). Briefly, the leaves of $P$. japonicus $(425.36 \mathrm{~g})$ were ground to fine particles using an electric mixer (HMF-3100 S; Hanil Electric, Seoul, Korea) for extraction at room temperature with $75 \% \mathrm{EtOH}$. The $\mathrm{EtOH}$ was then removed with a rotary evaporator and the remaining aqueous extract was fractionated consecutively with n-hexane, EtOAc, $\mathrm{BuOH}$, and water. The acquired hexane extract $(2.6728 \mathrm{~g})$ was evaporated in vacuum and the residue was used for silica gel $(40 \mu \mathrm{m}$; J.T. Baker; Thermo Fisher Scientific, Inc.) column chromatography $(100 \times 4.0 \mathrm{~cm})$ with a step gradient of $2.5,15$, and $25 \%$ acetone in dichloromethylene and 15 and $25 \% \mathrm{MeOH}$ in chloroform to obtain 62 fractions. Fraction 9 (MWLSH9; $304.9 \mathrm{mg}$ ) was separated on a Sephadex column $(100 \times 3.0 \mathrm{~cm})$ with $\mathrm{MeOH}$ as the eluent to obtain seven fractions. Fraction 3 (MWLSH9IC; 
$209.7 \mathrm{mg}$ ) was additionally separated on a Sephadex column $(100 \times 3.0 \mathrm{~cm})$ with $\mathrm{MeOH}$ to obtain five fractions. Fractions 2 and 3 (MWLSH9ICIB; $202.3 \mathrm{mg}$ ) were passed through a silica gel column $(100 \times 4.0 \mathrm{~cm})$ with $1.5 \%$ acetone in $\mathrm{CH}_{2} \mathrm{Cl}_{2}$ as the eluent to yield bakkenolide B $(173.8 \mathrm{mg})$. Pure bakkenolide B was identified using HPLC on a Phenomenex Luna C18 column (150 $4.6 \mathrm{~mm}$ ID; $5 \mu$ particle size; Phenomenex, Torrance, CA, USA) with an acetonitrile-water reagent alcohol gradient at a flow rate of $1.0 \mathrm{ml}$ per min. Bakkenolide B (Fig. 1A) isolated from $P$. japonicus leaves was identified using $1 \mathrm{H}, 13 \mathrm{C}$, and distortionless enhancement based on polarization transfer nuclear magnetic resonance spectroscopy in $\mathrm{CDCl} 3$, in contrast to previously reported spectral data (23).

Microglia culture. Mouse BV2 microglial cells, which were a generous gift from Professor Youn-Chul Kim at Wonkwang University (Iksan, Korea), were cultured in DMEM supplemented with $10 \%$ heat-inactivated fetal bovine serum at $37^{\circ} \mathrm{C}$ in a humidified atmosphere containing $5 \% \mathrm{CO}_{2}$. All experiments were performed with cells between passages 15 and 25 . Bakkenolide B was solubilized with DMSO at a final concentration of $0.1 \%$ and the stock solutions were directly added to the culture media.

Cell viability assay. The toxicity of bakkenolide B was assessed using an LDH leakage assay and an MTT reduction assay. Briefly, $5 \times 10^{4}$ cells were seeded in 24-well plates and allowed to grow to $80 \%$ confluence. MTT solution $(50 \mu \mathrm{g} / \mathrm{ml})$ was then added to each sample. Following incubation for $6 \mathrm{~h}$, the supernatant was removed, and the formazan crystals that formed in the normal cells were dissolved in DMSO. The absorbance of each sample was then measured at $570 \mathrm{~nm}$ using a Victor3 plate reader (PerkinElmer, Inc., Waltham, MA, USA). Extracellular LDH activity was determined using a cytotoxicity detection kit, according to the manufacturer's protocol. The absorbance in each well was quantified at $490 \mathrm{~nm}$ with a Victor3 plate reader (PerkinElmer, Inc.).

Measurement of $I L-1 \beta, I L-6, I L-12$ and TNF- $\alpha$ levels. IL-1 $\beta$, IL-6, IL-12 and TNF- $\alpha$ levels were quantified in the culture media using an ELISA kit (TNF- $\alpha$ cat. no. MTA00B; IL-1 $\beta$ cat. no. MLB00C; IL-6 cat. No M6000B and IL-12 cat. no. M1270) R\&D Systems, Inc.), according to the manufacturer's protocol.

Measurement of nitric oxide (NO) production. LPS $(1 \mu \mathrm{g} / \mathrm{ml})$ was added to microglia and incubated for $24 \mathrm{~h}$, following which the supernatant was obtained and mixed with Griess reagent, as previously reported (26). The absorbance of each mixture was then measured at $540 \mathrm{~nm}$ using a Victor3 plate reader (PerkinElmer, Inc.). The concentration of NO was determined using an NO standard.

Measurement of intracellular ROS. The intracellular ROS level, as an indicator of general oxidative stress, was estimated using the CM-H2DCFDA reagent. Briefly, $4 \times 10^{5}$ cells were seeded in 6-well plates and allowed to grow to $80 \%$ confluence. The cells were then harvested and washed thrice with phosphate buffer saline (PBS). A total of $10 \mu \mathrm{M}$ CM-H2DCFDA reagent was added to cells and incubated for $30 \mathrm{~min}$ in a $5 \% \mathrm{CO}_{2}$ at
A<smiles>C=C1COC(=O)[C@]12C[C@@]1(C)[C@@H](C)CC[C@H](OC(=O)/C(C)=C\C)[C@H]1[C@H]2OC(C)=O</smiles>

B

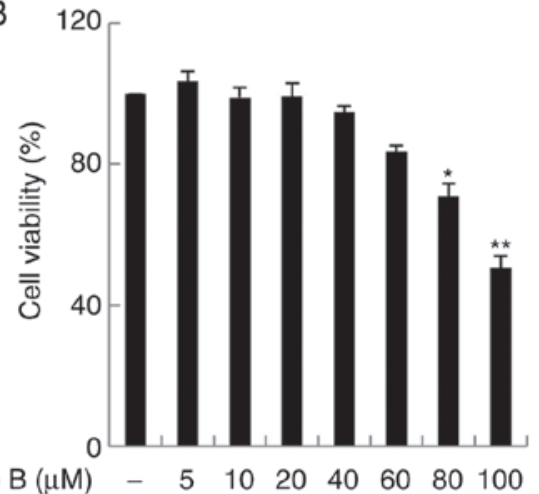

Bakkenolide B ( $\mu \mathrm{M}) \quad-\quad \begin{array}{lllllll}5 & 10 & 20 & 40 & 60 & 80 & 100\end{array}$

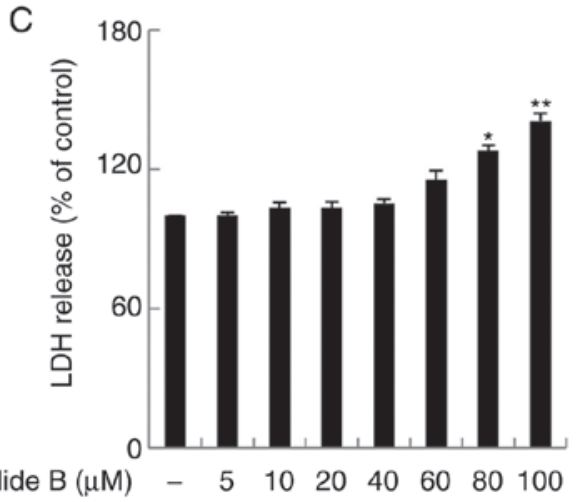

Figure 1. Cell viability of microglia cells treated with bakkenolide B. (A) Chemical structure of bakkenolide B. Microglia viability following bakkenolide B treatment was assessed using (B) MTT and (C) LDH assays. Microglia viability is expressed as the \% of the MTT or LDH value relative to the vehicle control (DMSO)-treated cells values (set at $100 \%$ ). ${ }^{*} \mathrm{P}<0.05$ and ${ }^{* *} \mathrm{P}<0.01$ compared with control. LDH, lactate dehydrogenase.

$37^{\circ} \mathrm{C}$. The fluorescence intensity was subsequently measured using a flow cytometer (Beckman Coulter, Inc., Brea, CA, USA). Finally, data were quantified using the CXP version 2.0 software (Beckman Coulter, Inc.). At least 10,000 cells were evaluated for each condition.

Gene silencing of murine Nrf2, HO-1 and NQO1. Knockdown of Nrf2, HO-1, and NQO1 using siRNA (10 nM) was achieved with the $\mathrm{X}$-tremeGENE siRNA transfection reagent kit, according to the manufacturer's protocols. Further experiments were performed $24 \mathrm{~h}$ following transfection. Nrf2, HO-1, NQO1, or control siRNA-transfected cells were used to measure the IL-1 $\beta$, IL-6, IL-12 and TNF- $\alpha$ levels.

Transient transfection and dual luciferase assay. Cells were transfected using the HO-1 promoter reporter plasmid or 

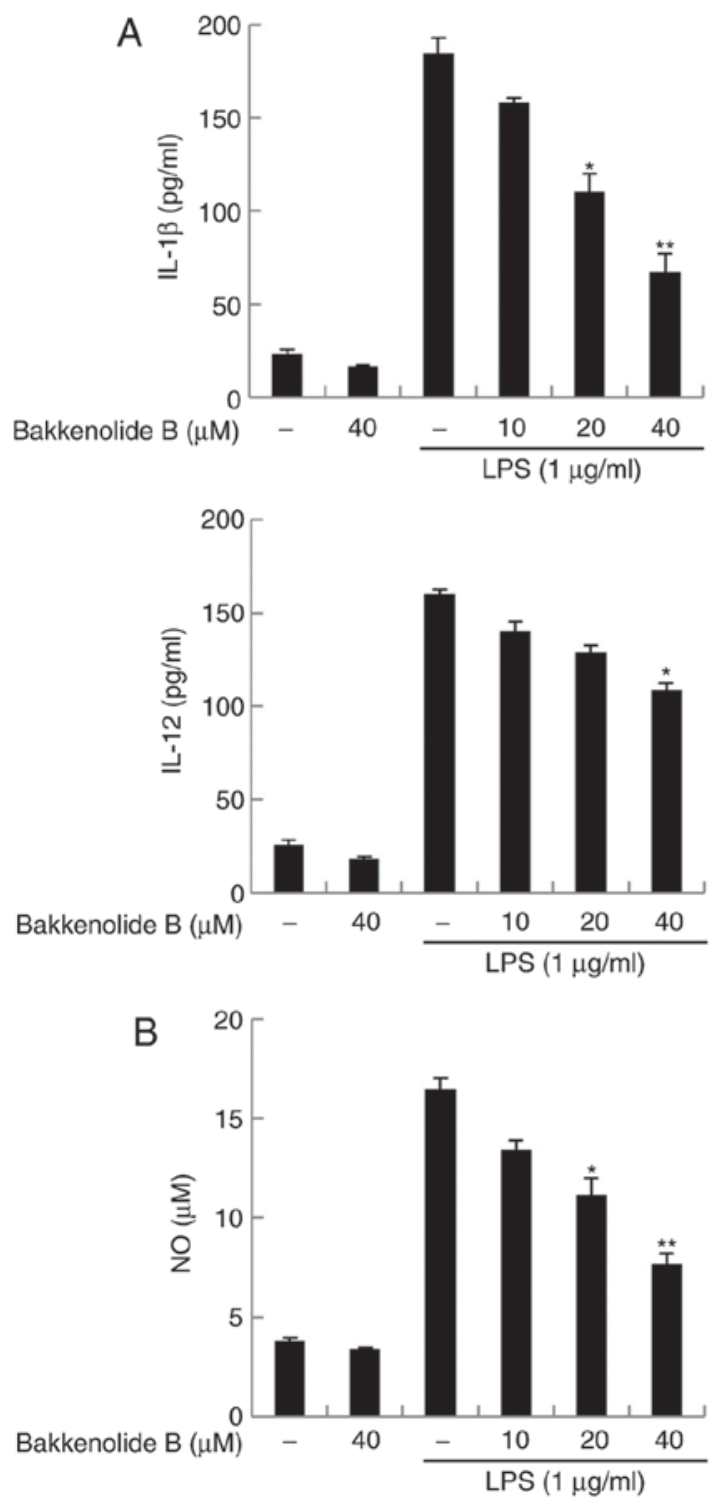
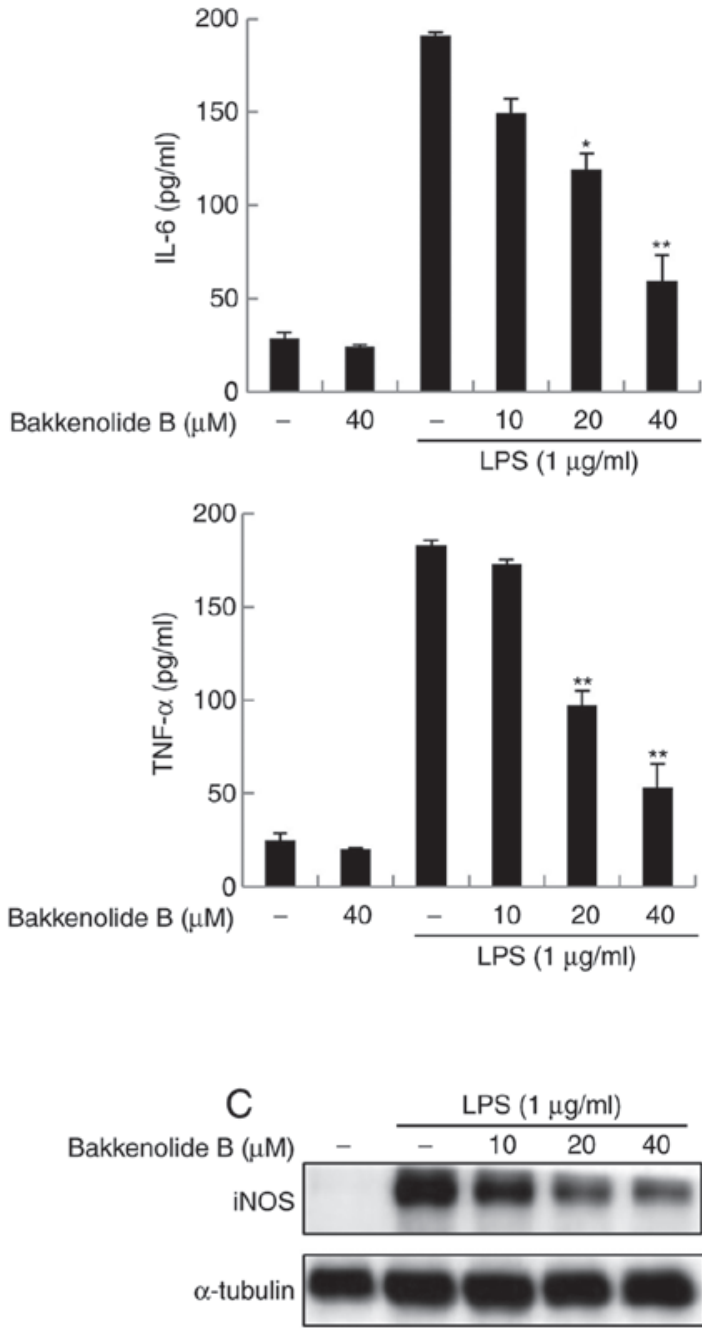

Figure 2. Bakkenolide B pretreatment inhibits the LPS-induced neuroinflammatory response. Microglia were pretreated with bakkenolide B for $1 \mathrm{~h}$, then exposed to LPS for $24 \mathrm{~h}$. (A) The concentrations of TNF- $\alpha$, IL-1 $\beta$, IL-6 and IL-12 in the culture media were measured using a commercial ELISA kit. (B) The production of NO was measured by the method of Griess. (C) Protein expression levels of iNOS were determined by western blotting. $\alpha$-tubulin was used as the internal loading control. All data are presented as the means \pm standard error of the mean $(n=3)$. ${ }^{*} \mathrm{P}<0.05$ and ${ }^{* *} \mathrm{P}<0.01$ compared with LPS alone group. LPS, lipopolysaccharide; TNF, tumor necrosis factor; IL, interleukin; NO, nitric oxide; iNOS, inducible nitric oxide synthase.

ARE reporter plasmids (100 ng; Agilent Technologies, Inc., Santa Clara, CA, USA) using FuGENE-HD reagent (Roche Applied Science, Penzberg, Germany), according to the manufacturer's protocol. A Renilla luciferase control plasmid was co-transfected as an internal control for transfection efficiency. Luciferase activity was examined using a dual-luciferase assay kit (Promega Corporation, Madison, IW, USA), according to the manufacturer's protocol. Luminescence was measured using a Victor3 plate reader (PerkinElmer, Inc.).

Western blot analysis. The cell were lysed with Radioimmunoprecipitation assay buffer(Thermo Fisher Scientific, Inc.) supplemented with a protease inhibitor cocktail. The nuclear extracts were prepared using the NE-PER nuclear and cytoplasmic extraction reagent (Thermo Fisher Scientific, Inc.) according to the manufacturer's protocol. Lysate protein concentrations were evaluated using Bio-Rad Protein Assay
Dye Reagent concentrate (Bio-Rad Laboratories, Hercules, CA, USA). Proteins in each sample (30-50 $\mu \mathrm{g}$ of total protein) were subjected to a $7.5-10 \%$ SDS-PAGE. and transferred to a polyvinylidene difluoride membrane (EMD Millipore, Billerica, MA, USA). The membranes were blocked with 5\% skimmed milk in PBS with $0.1 \%$ Tween-20 for $1 \mathrm{~h}$ at room temperature. The membrane was incubated with the primary antibodies ( $\alpha$-tubulin, TBP, iNOS, Nrf2, NQO1 and HO-1 at room temperature for $2 \mathrm{~h}$ and AMPK and p-AMPK at $4{ }^{\circ} \mathrm{C}$ overnight). Subsequently, the membranes were incubated with horseadish peroxidase conjugated seceondary antibodies (goat anti-rabbit, cat. no. sc-2004, 1:1,000; goat anto-mouse cat. no. sc-2039, 1:1,000) at room temperature for $1 \mathrm{~h}$. The membrane was visualized using a boosted chemiluminescent immunodetection system (Amersham; GEHealthcare, Chicago, IL, USA) and a secondary horseradish peroxidase-conjugated antibody. The data were quantified using an ImageQuant 350 
analyzer (ImageQuant TL SecurITy 8.0 software, Amersham; GE Healthcare).

Statistical analysis. Each experiment was repeated at least three times. Statistical analyses were conducted using the SPSS software version 18.0 (SPSS, Inc., Chicago, IL, USA). One-way analysis of variance followed by Dunn's post-hoc test was performed to identify differences among groups. $\mathrm{P}<0.05$ was considered to indicate a statistically significant difference.

\section{Results}

Bakkenolide B ameliorates neuroinflammatory cytokines in LPS-stimulated microglia. First, MTT and LDH assays were used to evaluate the viability of microglia exposed to bakkenolide B $(0-100 \mu \mathrm{M})$. The results demonstrated that bakkenolide $\mathrm{B}$ treatment at concentrations of 5-40 $\mu \mathrm{M}$ did not significantly affect microglia viability (Fig. 1B and C). Thus, in subsequent experiments, the microglia were evaluated following exposure to bakkenolide B at concentrations of 5-40 $\mu \mathrm{M}$. The anti-neuroinflammatory properties of bakkenolide B were then examined in LPS-stimulated microglia. Microglia exposed to LPS displayed increased IL-1 $\beta$, IL-6, IL-12 and TNF- $\alpha$ production compared with control cells exposed to vehicle. Conversely, bakkenolide B pretreatment reversed the effects of LPS on IL-1 $\beta$, IL-6, IL-12 and TNF- $\alpha$ production in a dose-dependent manner (Fig. 2A). The enzyme activity and protein expression of iNOS were also investigated, because this is known to be important for neuroinflammatory responses. Treatment with bakkenolide B significantly decreased NO production induced by LPS (Fig. 2B). In addition, pretreatment with bakkenolide B resulted in markedly decreased protein expression levels of iNOS (Fig. 2C). CM-H2DCFDA staining, which is a general oxidative stress indicator, was used to assess the impact of bakkenolide B pretreatment on LPS-stimulated microglia. The results demonstrated that LPS exposure increased ROS production to $23.5 \pm 4.3 \%$ in LPS-stimulated microglia compared to $5.8 \pm 0.1 \%$ in control untreated cells. Conversely, bakkenolide B pretreatment significantly decreased the LPS-induced ROS production (15.5 $\pm 1.7 \%$; Fig. 3). As expected, N-acetyl-L-cysteine (NAC; a ROS scavenger) significantly attenuated the LPS-induced ROS (Fig. 3). Taken together, these results suggest that bakkenolide B can inhibit the production of pro-neuroinflammatory cytokines and mediators in LPS-stimulated microglia.

Bakkenolide B activates Nrf2/ARE signaling and reduces neuroinflammation in LPS-stimulated microglia. To better understand the molecular pathway through which bakkenolide B inhibits the LPS-induced neuroinflammatory response, we evaluated whether Nrf2/ARE signaling is induced in bakkenolide B-treated microglia. First, the levels of nuclear Nrf2 accumulation activation were examined using western blotting and a luciferase promoter assay following treatment of microglia with bakkenolide B. As presented in Fig. 4A, nuclear Nrf2 accumulation rapidly increased $1 \mathrm{~h}$ following bakkenolide B treatment. Furthermore, bakkenolide B dose-dependently increased nuclear Nrf2 accumulation (Fig. 4A). Nuclear extracts were analyzed for the $\alpha$-tubulin
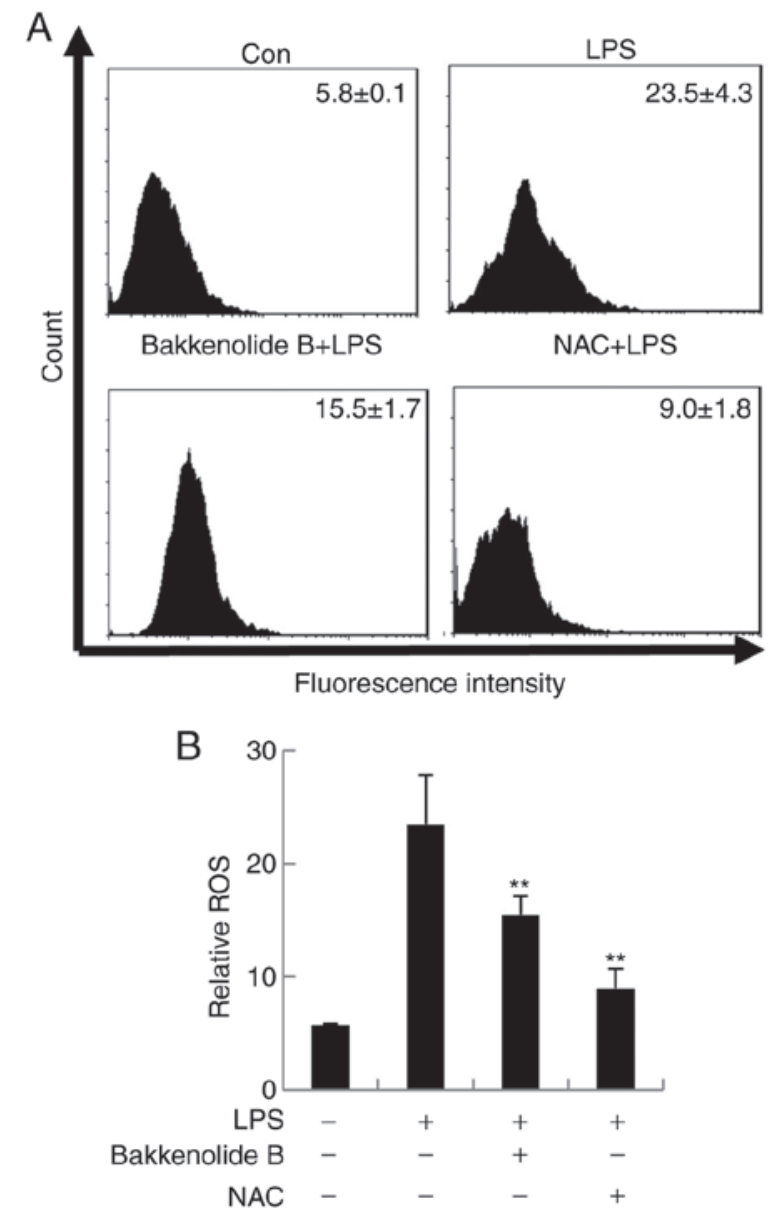

Figure 3. Bakkenolide B pretreatment inhibits the LPS-induced ROS production. Microglia were exposed to bakkenolide B $(40 \mu \mathrm{M})$ or NAC $(2 \mathrm{mM})$ for $1 \mathrm{~h}$, then treated with LPS for $1 \mathrm{~h}$. The ROS levels were measured using flow cytometry. (A) Representative plots of flow cytometry results. (B) Quantification of the relative \% of cells with ROS production. All data are presented as the means \pm standard error of the mean $(n=3)$. ${ }^{*} \mathrm{P}<0.05$ and ${ }^{* *} \mathrm{P}<0.01$ compared with LPS alone group. LPS, lipopolysaccharide; ROS, reactive oxygen species; NAC, N-acetyl-L-cysteine.

cytoplasmic marker and minimal cytoplasmic contamination was observed (Fig. 4A). Consistent with this result, bakkenolide B significantly induced the expression of HO-1 and NQO1 in microglia compared with controls, in a timeand dose-dependent manner (Fig. 4B). Bakkenolide B-treated microglia lysates were prepared to measure ARE promoter activity (based on luciferase activity normalized to renilla luciferase activity), and the results indicated that bakkenolide B dose-dependently increased ARE-promoter activity (Fig. 4C). Consistent with this finding, bakkenolide B also significantly increased the transcriptional activity of the HO-1 promoter in a dose-dependent manner (Fig. 4D). To test the hypothesis that the anti-neuroinflammatory properties of bakkenolide B are diminished following knockdown of Nrf2, HO-1 and NQO1, TNF- $\alpha$ levels were evaluated using a Quantikine ELISA kit following exposure of microglia to LPS. The results revealed that TNF- $\alpha$ production was markedly increased in LPS-stimulated cells transfected with the control siRNA, while exposure to bakkenolide B and the control siRNA significantly decreased TNF- $\alpha$ production compared with the LPS-only group (Fig. 4E). Notably, transfection of siRNA for Nrf2, 

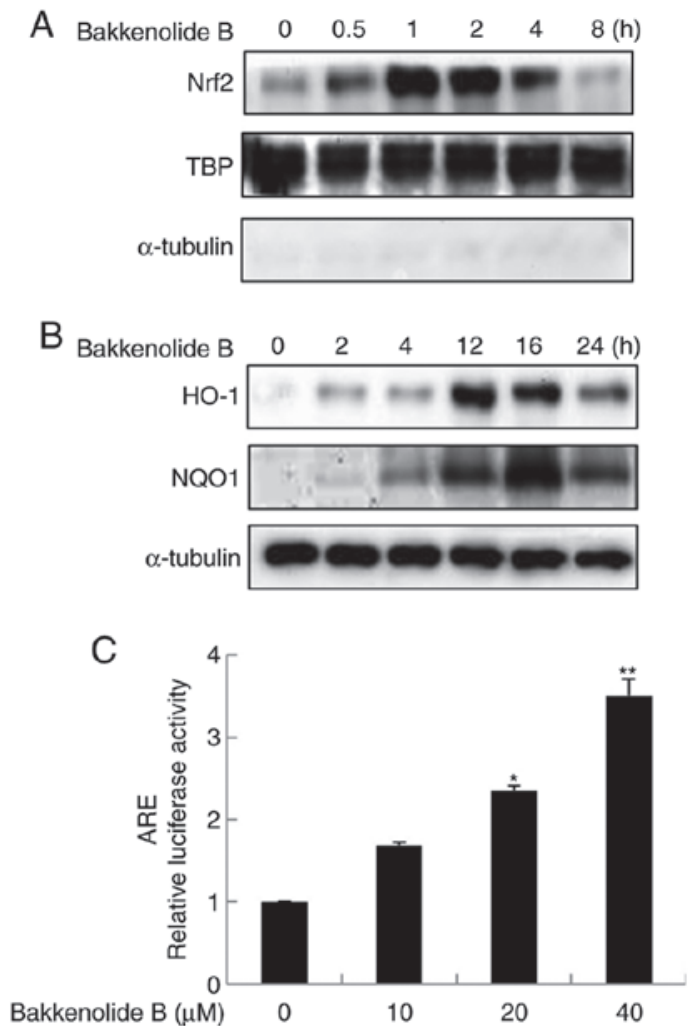
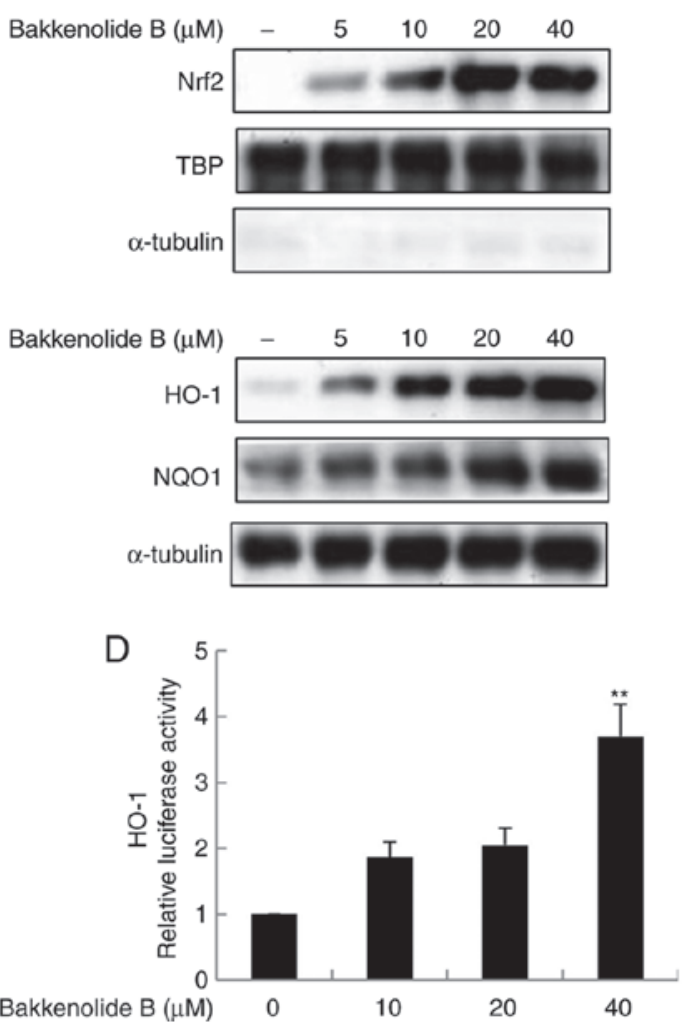

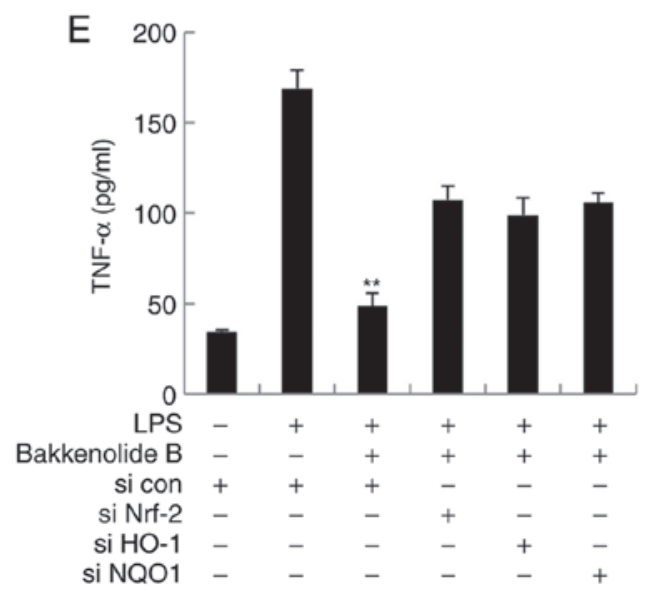

Figure 4. Bakkenolide B pretreatment suppresses the LPS-induced neuroinflammatory response via activation of Nrf2/ARE signaling. (A) The effects of bakkenolide B on Nrf2 nuclear accumulation were determined by western blotting. Microglia were incubated with $40 \mu \mathrm{M}$ bakkenolide B for different time periods $(0,0.5,1,2,4$ and $8 \mathrm{~h})$. Alternatively, cells were treated with different concentrations of bakkenolide B for $4 \mathrm{~h}$. Nuclear lysates were then collected, and the purity of nuclear extracts was confirmed by the absence of cytoplasmic $\alpha$-tubulin, while TBP was used as the internal loading control for nuclear protein. (B) Microglia were treated with bakkenolide B $(40 \mu \mathrm{M})$ for 4, 8, 12, 16 and $24 \mathrm{~h}$. Alternatively, the cells were treated with bakkenolide B at the indicated concentrations for $4 \mathrm{~h}$. Then the protein levels of HO-1 and NQO1 were measured by western blotting. $\alpha$-tubulin was used as an internal loading control. (C and D) Microglia were transfected with the ARE or HO-1 luciferase reporter plasmid, then incubated with bakkenolide B for $8 \mathrm{~h}$. Equal amounts of cell extract were assayed for dual-luciferase activity. " $\mathrm{P}<0.05$ and ${ }^{* * *} \mathrm{P}<0.01$ compared with control. (E) Microglia were transiently transfected with Nrf2, HO-1 or NQO1 siRNA or control siRNA and pretreated with bakkenolide B $(40 \mu \mathrm{M})$ for $1 \mathrm{~h}$, then exposed to LPS for $24 \mathrm{~h}$, and then analyzed using a TNF- $\alpha$ Quantikine ELISA kit. " $\mathrm{P}<0.05$ and ${ }^{* *} \mathrm{P}<0.01$ compared with LPS+control siRNA group. All data are presented as the means \pm standard error of the mean $(\mathrm{n}=3)$. LPS, lipopolysaccharide; Nrf2, nuclear factor erythroid 2-related factor 2; ARE, antioxidant response element; TBP, TATA binding protein; HO-1, heme oxygenase-1; NQO1, NADPH dehydrogenase quinone-1; si, small interfering; TNF, tumor necrosis factor.

HO-1 or NQO1 partially reversed the bakkenolide B-mediated inhibition of TNF- $\alpha$ production in LPS-stimulated microglia (Fig. 4E). These findings suggest that bakkenolide B may have significant anti-neuroinflammatory properties mediated by the Nrf2/ARE pathway.

Bakkenolide B-induced AMPK-mediated Nrf2/ARE activation is vital for the suppression of LPS-induced neuro- inflammation. To determine if AMPK signaling mediates bakkenolide B-induced Nrf2/ARE activation, the AMPK activation level were determined following bakkenolide B treatment in microglia using western blotting and antibodies targeting total and phosphorylated (at Thr 172) AMPK. As expected, bakkenolide B induced AMPK phosphorylation in a time- and dose-dependent manner (Fig. 5A and B). To determine if AMPK inhibition decreases Nrf2/ARE activation, 

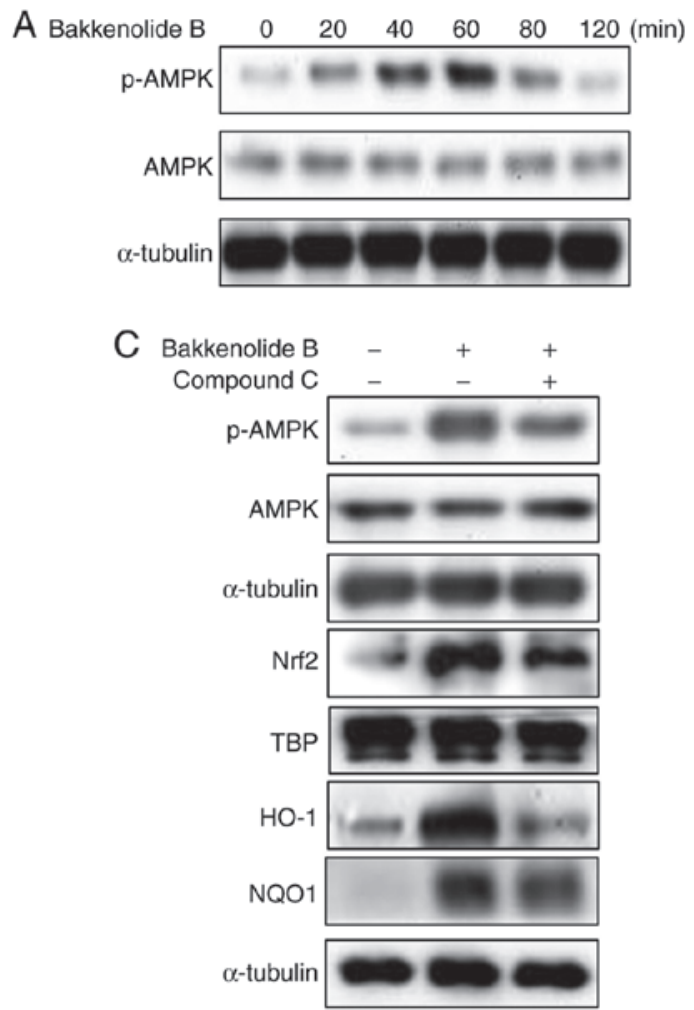
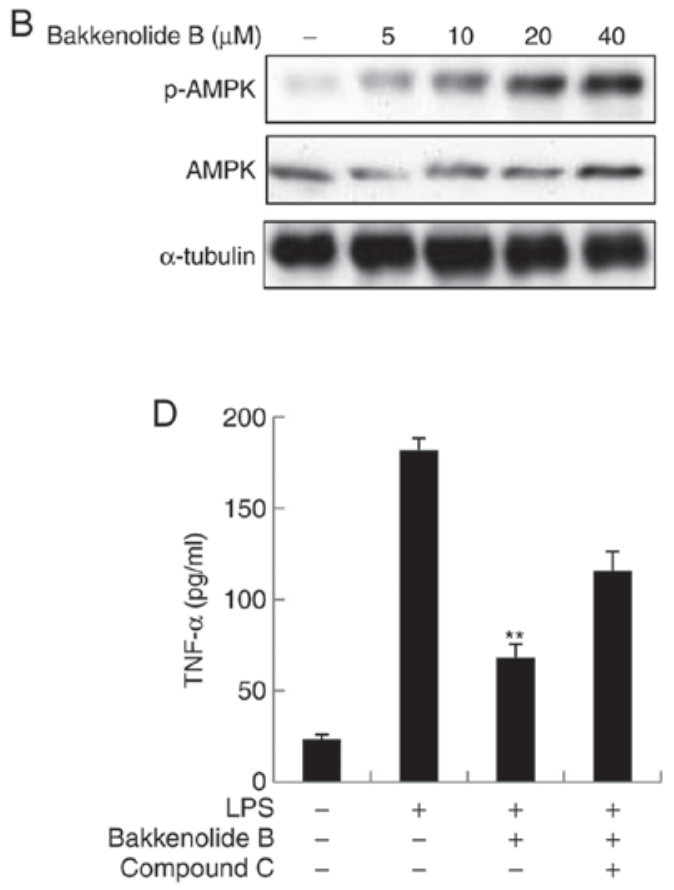

Figure 5. Anti-neuroinflammatory properties of bakkenolide B are regulated by AMPK. (A) Cells were treated with bakkenolide B (40 $\mu$ M) for the indicated times, following which the cell extracts were prepared for western blotting. (B) Cells were incubated with the indicated doses of bakkenolide B for $1 \mathrm{~h}$, following which the cell extracts were prepared for western blotting. (C) Cells were pretreated with $20 \mu \mathrm{M}$ compound C for $1 \mathrm{~h}$, then treated with bakkenolide B $(40 \mu \mathrm{M})$ for $1 \mathrm{~h}$. Total cell extracts were subjected to immunoblotting using anti-AMPK, Nrf2, HO-1 and NQO1 antibodies. (D) Cells were pretreated with $20 \mu \mathrm{M}$ compound $\mathrm{C}$ for $1 \mathrm{~h}$, then treated with bakkenolide B $(40 \mu \mathrm{M})$ for $1 \mathrm{~h}$, then exposed to LPS for $24 \mathrm{~h}$, and then analyzed using a TNF- $\alpha$ Quantikine ELISA kit. All data are presented as the means \pm standard error of the mean $(n=3) .{ }^{* *} \mathrm{P}<0.01$ compared with LPS alone group. AMPK, AMP-activated protein kinase; Nrf2, nuclear factor erythroid 2-related factor 2; HO-1, heme oxygenase-1; NQO1, NADPH dehydrogenase quinone-1; TNF, tumor necrosis factor; p, phosphorylated; TBP, TATA binding protein.

nuclear Nrf2 accumulation and protein expression of HO-1 and NQO1 were evaluated by western blot analysis. As expected, the AMPK inhibitor compound $\mathrm{C}$ significantly inhibited bakkenolide B-induced AMPK phosphorylation (Fig. 5C). Notably, AMPK inhibition decreased bakkenolide B-induced nuclear Nrf2 accumulation and protein expression of $\mathrm{HO}-1$ and NQO1 (Fig. 5C). To confirm that AMPK-mediated Nrf2/ARE signaling regulated the anti-neuroinflammatory properties of bakkenolide B protection against LPS-induced neuroinflammation, microglia were pretreated with compound $\mathrm{C}$ prior to exposure to bakkenolide B. Following treatment with bakkenolide B, microglia were exposed to LPS for $24 \mathrm{~h}$. As illustrated in Fig. 5D, compound C pretreatment partially reversed the bakkenolide B-mediated inhibition of TNF- $\alpha$ production. Taken together, these results suggest that AMPK activity is required for bakkenolide B-induced Nrf2 activation and subsequent anti-neuroinflammatory activity.

\section{Discussion}

The prevalence of neurodegenerative disorders has increased nowadays and has become one of the most serious health issues worldwide. Neurodegenerative disorders are associated with a higher risk of neurodegenerative diseases, including Huntington's, Alzheimer's and Parkinson's $(27,28)$. Abnormal microglia activation has a crucial role in the development of neurodegenerative disorders, although the exact mechanisms have not been fully elucidated. Limiting the production of neuroinflammatory mediators is crucial for neuronal protection and repair. Abnormal activation of the microglia and upregulation of pro-neuroinflammatory mediators are pathological features of neurodegenerative disorders (29-31). Furthermore, abnormal microglial accumulation and release of neuroinflammatory mediators is deleterious to neighboring neurons and may be related to neurodegenerative disease (32). The current study presents several major findings. First, bakkenolide B was demonstrated to have the potential for use as a treatment to ameliorate abnormal neuroinflammatory responses. Second, AMPK/Nrf2/ARE signaling was demonstrated to have a significant role in the anti-neuroinflammatory activities of bakkenolide B. The present study is the first to demonstrate the anti-neuroinflammatory mechanisms that regulate the effects of bakkenolide B in LPS-stimulated microglia.

Neuronal injury results from ongoing abnormal microglia activation or deficient/suppressed neuron recovery. Abnormal microglia activation contributes to the development of neurodegenerative disorder, and recent evidence has highlighted the anti-neuroinflammatory effects in microglia, providing novel potential avenues for treatment of neurodegenerative diseases (33). These proinflammatory cytokines, including TNF- $\alpha$, IL-1 $\beta$, IL- 6 and IL-12, are secreted from microglia and initiate, amplify, and perpetuate the neuroinflammatory 

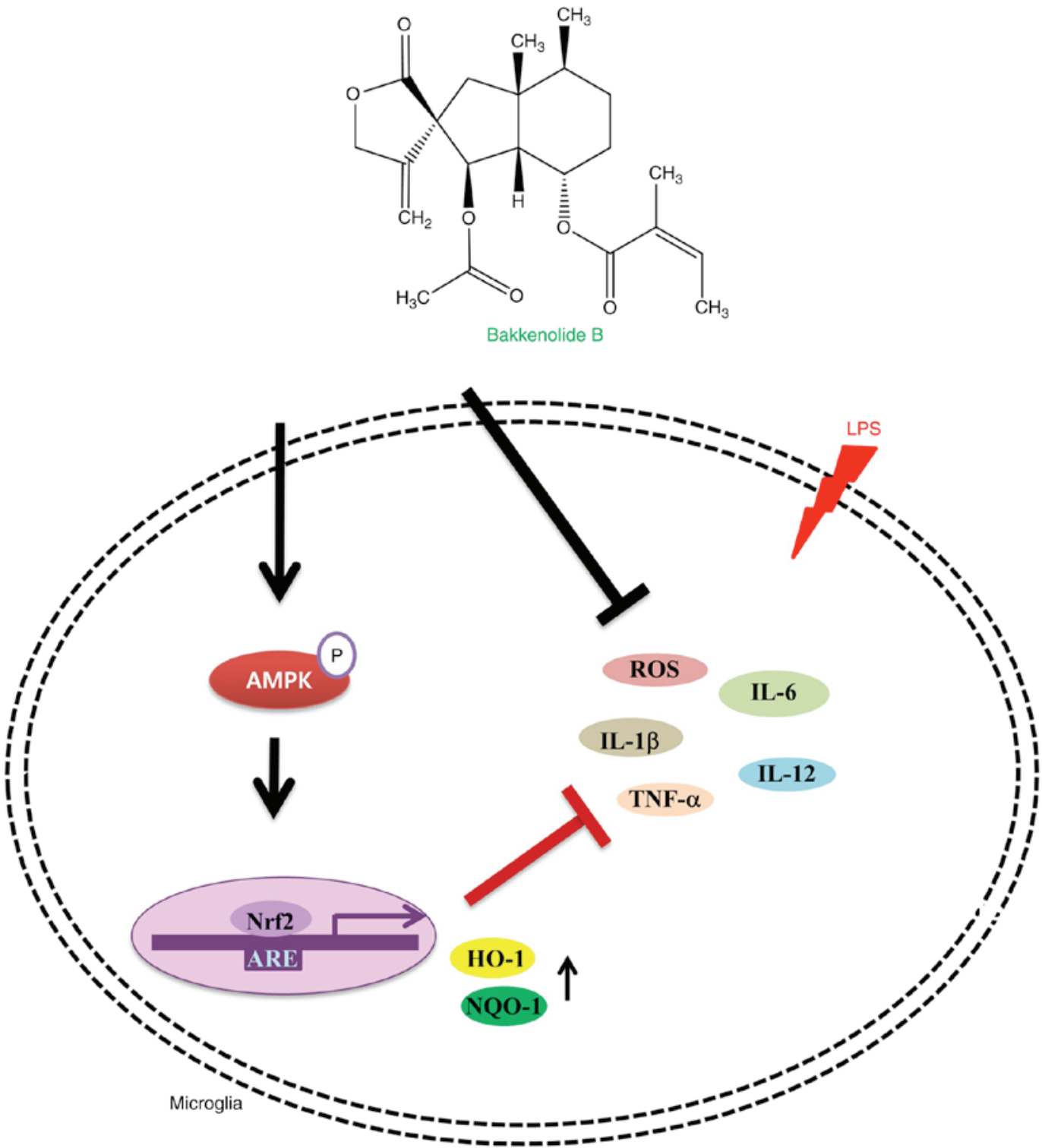

Figure 6. Schematic of the proposed mechanism for the anti-neuroinflammatory properties of bakkenolide B in LPS-stimulated microglia.

response in the brain. These cytokines are the primary endogenous mediators of the neuroinflammatory reaction, causing not only abnormal microglia activation, but also triggering damage to adjacent neurons $(34,35)$. The present study demonstrated that following LPS exposure, IL-1 $\beta$, IL-6, IL-12 and TNF- $\alpha$ in microglia are significantly increased. However, pretreatment with bakkenolide B significantly reduced the levels of IL-1 $\beta$, IL-6, IL-12 and TNF- $\alpha$ in microglia. ROS were also induced via inflammatory mediators during the neuroinflammatory response in microglia, but this LPS-induced production of ROS was diminished following bakkenolide B pretreatment. These results demonstrated that the anti-neuroinflammatory properties of bakkenolide B in microglia may be attributed to the inhibition of IL-1 $\beta$, IL-6, IL-12 and TNF- $\alpha$, and ROS production.

Nrf2/ARE signaling in microglia is essential to reducing neuronal cell death and preserving cognitive function. Studies of microglia have reported that HO-1 and NQO1 overexpression, mediated by Nrf2 activation, has a strong anti-neuroinflammatory effect (11). This finding has been observed in both cell culture and animal models treated with different natural compounds that activate Nrf2/ARE to reduce the LPS-induced pro-inflammatory response and inhibit oxidative damage. Natural compounds exhibit both antioxidant and anti-inflammatory effects depending on the Nrf2/ARE signal that prevents neurodegeneration. Several studies have reported that phase II antioxidant enzymes may contribute to neural deterioration in neurodegenerative disorders and diseases. Remarkably, Nrf-2 signaling activates phase II antioxidant enzymes, such as HO-1 and NQO-1 $(12,13)$. These enzymes serve crucial roles in neurodegenerative disorders via abnormal neuroinflammatory responses. The present results also indicate that bakkenolide B-induced Nrf2 activation upregulated HO-1 and NOQ1 expression and consequently diminished LPS-mediated neuroinflammatory responses.

AMPK is an essential factor in the protein kinase cascade that has a vital role in regulation of energy metabolism, which is important for the regulation of cell homeostasis. A previous 
study has reported that the molecular mechanisms and signaling pathways regulating AMPK signal activation that mediate its neuroprotective effects are driven by its anti-neuroinflammatory activities (36). Furthermore, the neuroprotective action of AMPK may not only be mediated by its antioxidant and anti-inflammatory action, but also due to the enhancement of Nrf2/ARE signaling. Indeed, studies have demonstrated the involvement of molecules downstream of AMPK in the modulation of Nrf2 activation and HO-1 and NQO-1 expression in neurons and microglia $(18,19)$. More recently, the role of AMPK in the induction of neuroprotective factors has been confirmed in mice that show improved recovery following stroke in response to AMPK-dependent Nrf2/ARE signaling activation in the hippocampus (37). Thus, Nrf2/ARE activation has been demonstrated to be neuroprotective. Consistent with this finding, the present study demonstrated that the anti-neuroinflammatory properties of bakkenolide $\mathrm{B}$ were attributed to the activation of AMPK/Nrf2/ARE signaling. Bakkenolide B-mediated AMPK signaling was critical in Nrf2/ARE activation, which subsequently suppressed LPS-mediated neuroinflammatory responses. These results suggest that bakkenolide B may reduce LPS-stimulated neuroinflammatory responses by inducing the AMPK/Nrf2/ARE signaling pathway.

In conclusion, this is the first study linking bakkenolide B, isolated from P. japonicus, with AMPK signaling. These findings are especially important because AMPK mediates activation of Nrf2, HO-1 and NQO1. Bakkenolide B mediated the activation of AMPK, subsequently induced nuclear Nrf2 translocation, and promoted Nrf-2-mediated HO-1 and NQO1 expression, leading to attenuation of LPS-stimulated neuroinflammatory response (Fig. 6). Overall, these results suggest that bakkenolide $\mathrm{B}$ exposure may be a promising therapeutic strategy to protect against abnormal microglia activation in neurodegenerative diseases.

\section{Acknowledgements}

This study was supported by the Basic Science Research Program through the National Research Foundation of Korea funded by the Ministry of Education (grant nos. NRF-2015R1D1A1A01059450 and NRF-2016R1D1A3B03934083).

\section{References}

1. Letiembre M, Liu Y, Walter S, Hao W, Pfander T, Wrede A, Schulz-Schaeffer W and Fassbender K: Screening of innate immune receptors in neurodegenerative diseases: A similar pattern. Neurobiol Aging 30: 759-768, 2009.

2. Frank-Cannon TC, Alto LT, McAlpine FE and Tansey MG: Does neuroinflammation fan the flame in neurodegenerative diseases? Mol Neurodegener 4: 47, 2009.

3. Mueller AM, Yoon BH and Sadiq SA: Inhibition of hyaluronan synthesis protects against central nervous system (CNS) autoimmunity and increases CXCL12 expression in the inflamed CNS. J Biol Chem 289: 22888-22899, 2014.

4. Slusarczyk J, Trojan E, Glombik K, Piotrowska A, Budziszewska B, Kubera M, Popiolek-Barczyk K, Lason W, Mika J and Basta-Kaim A: Anti-inflammatory properties of tianeptine on lipopolysaccharide-induced changes in microglial cells involve toll-like receptor-related pathways. J Neurochem 136: 958-970, 2016.

5. Tao L, Zhang F, Hao L, Wu J, Jia J, Liu JY, Zheng LT and Zhen X: 1-O-tigloyl-1-O-deacetyl-nimbolinin B inhibits LPS-stimulated inflammatory responses by suppressing NF-kappaB and JNK activation in microglia cells. J Pharmacol Sci 125: 364-374, 2014
6. Ransohoff RM, Schafer D, Vincent A, Blachère NE and Bar-Or A: Neuroinflammation: Ways in which the immune system affects the brain. Neurotherapeutics 12: 896-909, 2015.

7. Jebelli J, Hooper C and Pocock JM: Microglial p53 activation is detrimental to neuronal synapses during activation-induced inflammation: Implications for neurodegeneration. Neurosci Lett 583: 92-97, 2014.

8. Xia CY, Zhang S, Gao Y, Wang ZZ and Chen NH: Selective modulation of microglia polarization to M2 phenotype for stroke treatment. Int Immunopharmacol 25: 377-382, 2015.

9. Gaire BP, Kwon OW, Park SH, Chun KH, Kim SY, Shin DY and Choi JW: Neuroprotective effect of 6-paradol in focal cerebral ischemia involves the attenuation of neuroinflammatory responses in activated microglia. PLoS One 10: e0120203, 2015.

10. Lee JA, Kim JH, Woo SY, Son HJ, Han SH, Jang BK, Choi JW, Kim DJ, Park KD and Hwang O: A novel compound VSC2 has anti-inflammatory and antioxidant properties in microglia and in parkinson's disease animal model. Br J Pharmacol 172: 1087-100, 2015.

11. Mazzuferi M, Kumar G, van Eyll J, Danis B, Foerch P and Kaminski RM: Nrf2 defense pathway: Experimental evidence for its protective role in epilepsy. Ann Neurol 74: 560-568, 2013.

12. Onasanwo SA, Velagapudi R, El-Bakoush A and Olajide OA: Inhibition of neuroinflammation in BV2 microglia by the biflavonoid kolaviron is dependent on the Nrf2/ARE antioxidant protective mechanism. Mol Cell Biochem 414: 23-36, 2016.

13. Jayasooriya RG, Lee KT, Choi YH, Moon SK, Kim WJ and Kim GY: Antagonistic effects of acetylshikonin on LPS-induced NO and PGE2 production in BV2 microglial cells via inhibition of ROS/PI3K/akt-mediated NF-kappaB signaling and activation of Nrf2-dependent HO-1. In Vitro Cell Dev Biol Anim 51: 975-986, 2015.

14. Chen J, Yin W, Tu Y, Wang S, Yang X, Chen Q, Zhang X, Han Y and Pi R: L-F001, a novel multifunctional ROCK inhibitor, suppresses neuroinflammation in vitro and in vivo: Involvement of $\mathrm{NF}-\kappa \mathrm{B}$ inhibition and Nrf2 pathway activation. Eur J Pharmacol 806: 1-9, 2017.

15. Ismaiel AA, Espinosa-Oliva AM, Santiago M, GarcíaQuintanilla A, Oliva-Martín MJ, Herrera AJ, Venero JL and de Pablos RM: Metformin, besides exhibiting strong in vivo anti-inflammatory properties, increases mptp-induced damage to the nigrostriatal dopaminergic system. Toxicol Appl Pharmacol 298: 19-30, 2016.

16. Syed Hussein SS, Kamarudin MN and Kadir HA: (+)-Catechin attenuates NF-kappaB activation through regulation of akt, MAPK, and AMPK signaling pathways in LPS-induced BV-2 microglial cells. Am J Chin Med 43: 927-952, 2015.

17. Xu Y, Xu Y, Wang Y, Wang Y, He L, Jiang Z, Huang Z, Liao H, Li J, Saavedra JM, et al: Telmisartan prevention of LPS-induced microglia activation involves M2 microglia polarization via CaMKK $\beta$-dependent AMPK activation. Brain Behav Immun 50: 298-313, 2015.

18. Lee YY, Park JS, Lee EJ, Lee SY, Kim DH, Kang JL and Kim HS: Anti-inflammatory mechanism of ginseng saponin metabolite $\mathrm{Rh} 3$ in lipopolysaccharide-stimulated microglia: Critical role of 5'-adenosine monophosphate-activated protein kinase signaling pathway. J Agric Food Chem 63: 3472-3480, 2015.

19. Park SY, Jin ML, Wang Z, Park G and Choi YW: 2,3,4',5-tetrahyd roxystilbene-2-O- $\beta$-d-glucoside exerts anti-inflammatory effects on lipopolysaccharide-stimulated microglia by inhibiting NF- $\kappa \mathrm{B}$ and activating AMPK/Nrf2 pathways. Food Chem Toxicol 97: 159-167, 2016.

20. Zhou X, Cao Y, Ao G, Hu L, Liu H, Wu J, Wang X, Jin M, Zheng S, Zhen X, et al: CaMKK $\beta$-dependent activation of AMP-activated protein kinase is critical to suppressive effects of hydrogen sulfide on neuroinflammation. Antioxid Redox Signal 21: 1741-1758, 2014.

21. Dong XW, Li RJ, Gao X and Row KH: Bakkenolides from petasites tatewakianus. Fitoterapia 81: 153-156, 2010.

22. Zhang FJ, Wang Q, Wang Y and Guo ML: Anti-allergic effects of total bakkenolides from petasites tricholobus in ovalbumin-sensitized rats. Phytother Res 25: 116-121, 2011.

23. Lee KP, Kang S, Park SJ, Choi YW, Lee YG and Im DS: Anti-allergic and anti-inflammatory effects of bakkenolide B isolated from petasites japonicus leaves. J Ethnopharmacol 148: 890-894, 2013.

24. Wang S, Jin DQ, Xie C, Wang H, Wang M, Xu J and Guo Y: Isolation, characterization, and neuroprotective activities of sesquiterpenes from petasites japonicus. Food Chem 141: 2075-2082, 2013. 
25. Xu J, Yang B, Guo Y, Jin DQ, Guo P, Liu C, Hou W, Zhang T, Gui L and Sun Z: Neuroprotective bakkenolides from the roots of valeriana jatamansi. Fitoterapia 82: 849-853, 2011.

26. Lee YJ, Park SY, Kim SG, Park DJ, Kang JS, Lee SJ, Yoon S, Kim YH, Bae YS and Choi YW: Identification of a novel compound that inhibits iNOS and COX-2 expression in LPS-stimulated macrophages from schisandra chinensis. Biochem Biophys Res Commun 391: 1687-1692, 2010.

27. Fan H, Wu PF, Zhang L, Hu ZL, Wang W, Guan XL, Luo H, Ni M, Yang JW, Li MX, et al: Methionine sulfoxide reductase A negatively controls microglia-mediated neuroinflammation via inhibiting ROS/MAPKs/NF- $\kappa \mathrm{B}$ signaling pathways through a catalytic antioxidant function. Antioxid Redox Signal 22: 832-847, 2015

28. Song SY, Jung YY, Hwang CJ, Lee HP, Sok CH Kim JH, Lee SM, Seo HO, Hyun BK, Choi DY, et al: Inhibitory effect of ent-sauchinone on amyloidogenesis via inhibition of STAT3-mediated NF- $\kappa \mathrm{B}$ activation in cultured astrocytes and microglial BV-2 cells. J Neuroinflammation 11: 118, 2014.

29. Wu LH, Lin C, Lin HY, Liu YS, Wu CY, Tsai CF, Chang PC, Yeh WL and Lu DY: Naringenin suppresses neuroinflammatory responses through inducing suppressor of cytokine signaling 3 expression. Mol Neurobiol 53: 1080-1091, 2016.

30. Kang SM, More SV, Park JY, Kim BW, In PJ, Yoon SH and Choi DK: A novel synthetic HTB derivative, BECT inhibits lipopolysaccharide-mediated inflammatory response by suppressing the p38 MAPK/JNK and NF- $\mathrm{KB}$ activation pathways. Pharmacol Rep 66: 471-479, 2014.

31. Santa-Cecilia FV, Socias B, Ouidja MO, Sepulveda-Diaz JE Acuña L, Silva RL, Michel PP, Del-Bel E, Cunha TM and Raisman-Vozari R: Doxycycline suppresses microglial activation by inhibiting the p38 MAPK and NF- $\kappa \mathrm{B}$ signaling pathways. Neurotox Res 29: 447-459, 2016.
32. Vinoth Kumar R, Oh TW and Park YK: Anti-inflammatory effects of ginsenoside-Rh2 inhibits LPS-induced activation of microglia and overproduction of inflammatory mediators via modulation of TGF- $\beta 1 /$ smad pathway. Neurochem Res 41: 951-957, 2016.

33. Jeong YH, Kim Y, Song H, Chung YS, Park SB and Kim HS: Anti-inflammatory effects of $\alpha$-galactosylceramide analogs in activated microglia: Involvement of the p38 MAPK signaling pathway. PLoS One 9: e87030, 2014.

34. Shu Z, Yang B, Zhao H, Xu B, Jiao W, Wang Q, Wang Z and, Kuang H: Tangeretin exerts anti-neuroinflammatory effects via $\mathrm{NF}-\mathrm{KB}$ modulation in lipopolysaccharide-stimulated microglial cells. Int Immunopharmacol 19: 275-282, 2014.

35. Tanaka T, Kai S, Matsuyama T, Adachi T, Fukuda K and Hirota K: General anesthetics inhibit LPS-induced IL-1 $\beta$ expression in glial cells. PLoS One 8: e82930, 2013.

36. Lin HY, Huang BR, Yeh WL, Lee $\mathrm{CH}$, Huang SS, Lai $\mathrm{CH}$, Lin $\mathrm{H}$ and Lu DY: Antineuroinflammatory effects of lycopene via activation of adenosine monophosphate-activated protein kinase-alpha1/heme oxygenase-1 pathways. Neurobiol Aging 35: 191-202, 2014.

37. Ashabi G, Khalaj L, Khodagholi F, Goudarzvand M and Sarkaki A: Pre-treatment with metformin activates Nrf2 antioxidant pathways and inhibits inflammatory responses through induction of AMPK after transient global cerebral ischemia. Metab Brain Dis 30: 747-754, 2015. 